\title{
On some properties of $L B$-splines
}

\author{
by ZygMUNT Wronicz (Kraków)
}

\section{Franciszek Leja in memoriam}

\begin{abstract}
We give a relation between basic splines in the spaces of Tchebyshev splines and polynomial splines. By means of a new definition of normalized basic splines in the space of Tchebyshev splines we obtain a few further propreties of basic splines analogous to polynomial splines and we apply the results obtained to $L$-splines.
\end{abstract}

1. Introduction. The purpose of this paper is to generalize some properties of polynomial $B$-splines to basic splines in the space of Tchebyshevian spline functions, to obtain a relation between those basic splines and then to apply it to generalized splines.

Further, we need the following notations, definitions and properties of extended complete Tchebyshev systems (ECT-systems, see [5]).

Definition 1. We say that $\left\{u_{i}\right\}_{i=0}^{n}, u_{i} \in C^{n}[a, b]$ is an ECT-system on $[a, b]$ if, for any points $a \leqslant t_{0} \leqslant t_{1} \leqslant \ldots \leqslant t_{k} \leqslant b, k=0, \ldots, n$,

$$
D\left(\begin{array}{l}
t_{0}, \ldots, t_{k} \\
u_{0}, \ldots, u_{k}
\end{array}\right)=\operatorname{det}\left(D^{d_{j}} u_{i}\left(t_{j}\right)\right)_{i, j=0}^{k}>0,
$$

where $d_{j}=\max \left\{l: t_{j}=t_{j-1}=\ldots=t_{j-l}\right\}, j=0, \ldots, k$, and $D$ is the differentiation operator.

An ECT-system $\left\{u_{i}\right\}_{0}^{n}, u_{0}=1$ admits the representation

$$
\begin{aligned}
& u_{0}(t)=1 \text {, } \\
& u_{1}(t)=\int_{a}^{t} w_{1}\left(\tau_{1}\right) d \tau_{1}, \\
& u_{2}(t)=\int_{a}^{t} w_{1}\left(\tau_{1}\right) \int_{a}^{\tau_{1}} w_{2}\left(\tau_{2}\right) d \tau_{2} d \tau_{1}, \\
& u_{n}(t)=\int_{a}^{t} w_{1}\left(\tau_{1}\right) \int_{a}^{\tau_{1}} w_{2}\left(\tau_{2}\right) \ldots \int_{a}^{\tau_{n}-1} w_{n}\left(\tau_{n}\right) d \tau_{n} \ldots d \tau_{1},
\end{aligned}
$$

where $w_{i} \in C^{n-i}[a, b]$ and $w_{i}(t)>0$ for $t \in[a, b], i=0, \ldots, n$. 
The adjoint system $\left\{v_{i}\right\}_{0}^{n}$ is defined as follows:

$$
\begin{aligned}
& v_{0}(t)=1, \\
& v_{1}(t)=\int_{a}^{t} w_{n}\left(\tau_{1}\right) d \tau_{1}, \\
& v_{2}(t)=\int_{a}^{t} w_{n}\left(\tau_{1}\right) \int_{a}^{\tau_{1}} w_{n-1}\left(\tau_{2}\right) d \tau_{2} d \tau_{1}, \\
& \ldots \ldots \ldots \ldots \ldots \ldots \ldots \ldots \ldots \ldots \ldots \\
& v_{n}(t)=\int_{a}^{t} w_{n}\left(\tau_{1}\right) \int_{a}^{\tau_{1}} w_{n-1}\left(\tau_{2}\right) \ldots \int_{a} w_{1}\left(\tau_{n}\right) d \tau_{n} \ldots d \tau_{1} .
\end{aligned}
$$

Define

$$
\begin{aligned}
D_{j} \varphi & =\frac{1}{w_{j}} \frac{d}{d t} \varphi, & D_{j}^{*} \varphi & =\frac{d}{d t}\left(\frac{\varphi}{w_{j}}\right), \\
L_{j} \varphi & =D_{0} D_{j} \ldots D_{1} \varphi, & L_{j}^{*} \varphi & =D_{1}^{*} \ldots D_{j}^{*} D_{0}^{*} \varphi, \quad j=0, \ldots, n .
\end{aligned}
$$

Systems (1) and (2) span the null spaces of the differential operators $L_{n}$ and $L_{n}^{*}$, respectively.

Let $\Delta=\left\{a=t_{-n}=\ldots=t_{0}<t_{1}=\ldots=t_{\alpha_{1}}<t_{a_{1}+1}=\ldots=t_{\alpha_{1}+a_{2}}<\ldots\right.$ $\left.<t_{N}=\ldots=t_{N+n}=b\right\}=\left\{a=s_{0}<s_{1}<\ldots<s_{M}=b\right\} ; \alpha_{j}$ is the multiplicity of the point $s_{j}, j=0, \ldots, M, \alpha_{0}=\alpha_{M}=n+1, \sum_{j=0}^{M} \alpha_{j}=N+2 n+1$.

Definition 2. A function $s$ is called a Tchebyshev spline function w.r.t. the operator $L_{n}$ and the partition $\Delta$ if

(a) $L_{n} s=0$ in the intervals $\left(s_{j-1}, s_{j}\right), j=1, \ldots, M$,

(b) $\exists \varepsilon_{j}>0: s \subset C^{n-\alpha_{j}}\left(s_{j}-\varepsilon_{j}, s_{j}+\varepsilon_{j}\right), j=1, \ldots, M-1$.

We denote the set of these functions by $S_{\Delta}^{n}[a, b]$.

Lemma 1 (see [5]). The function

$$
\varphi_{n}(t, x)= \begin{cases}\int_{x}^{t} w_{n}\left(\tau_{1}\right) \int_{x}^{\tau_{1}} w_{n-1}\left(\tau_{2}\right) \ldots \int_{x}^{\tau_{n-1}} w_{1}\left(\tau_{n}\right) d \tau_{n} \ldots d \tau_{1} & \text { for } x \leqslant t \leqslant b, \\ 0 & \text { for } t<x,\end{cases}
$$

satisfies the following condition:

$$
D\left(\begin{array}{l}
t_{0}, \ldots, t_{n}, t_{n+1} \\
v_{0}, \ldots, v_{n}, \varphi_{n}
\end{array}\right) \geqslant 0
$$

for any system $\left\{t_{j}\right\}_{0}^{n+1}$ such that $a \leqslant t_{0} \leqslant \ldots \leqslant t_{n+1} \leqslant b$.

For $n=0$

$$
\varphi_{n}(t, x)= \begin{cases}1 & \text { for } x \leqslant t \leqslant b \\ 0 & \text { for } t<x\end{cases}
$$


Further, for $a \leqslant x \leqslant t_{0} \leqslant \ldots \leqslant t_{n+1} \leqslant b$

$$
D\left(\begin{array}{l}
t_{0}, \ldots, t_{n}, t_{n+1} \\
v_{0}, \ldots, v_{n}, \varphi_{n}
\end{array}\right)=0
$$

and for $t_{0}<x<t_{n+1}$

$$
D\left(\begin{array}{l}
t_{0}, \ldots, t_{n} t_{n+1} \\
v_{0}, \ldots, v_{n}, \varphi_{n}
\end{array}\right)>0 .
$$

The function $\varphi_{n}$ is a Green function associated with the operator $L_{n}^{*}$ and the function $w(t)=\int_{a}^{b} \varphi_{n}(t, x) d x$ is a solution of the equation $L_{n}^{*} w=1$.

Definition 3. We define the divided difference of a function $f$ at the points $t_{0} \leqslant \ldots \leqslant t_{n+1}, t_{0}<t_{n+1}$ w.r.t. the operator $L_{n}^{*}$ by

$$
\left[t_{0}, \ldots, t_{n+1} ; f\right]_{*}=\frac{D\left(\begin{array}{l}
t_{0}, \ldots, t_{n}, t_{n+1} \\
v_{0}, \ldots, v_{n}, f
\end{array}\right)}{D\left(\begin{array}{l}
t_{0}, \ldots, t_{n}, t_{n+1} \\
v_{0}, \ldots, v_{n}, w
\end{array}\right)}
$$

Remark. For the system $\left\{t^{i}\right\}_{0}^{n}, w_{0}=1, w_{i}=i, i=1, \ldots, n$, and $\left[t_{0}, \ldots, t_{n+1} ; f\right]_{*}=(n+1)\left[t_{0}, \ldots, t_{n+1} ; f\right]$, where the last expresion is the usual divided difference (see [3]). Properties of generalized divided differences may be found in [4] and [7].

Definition 4. The $i$ th $L_{n} B$-spline ( $B$-spline w.r.t. the system $\left\{u_{i}\right\}_{0}^{n}$ ) w.r.t. the partition $\Delta$ is defined by

$$
M_{i, n}(x)=\left[t_{i}, \ldots, t_{i+n+1} ; \varphi_{n}(t, x)\right]_{*}, \quad i=-n, \ldots, N-1 .
$$

We can see that

$$
\frac{d}{d x} \varphi_{n}(t, x)=-w_{1}(x) \varphi_{n-1}(t, x),
$$

where

$$
\begin{aligned}
& \varphi_{n-1}(t, x) \\
& = \begin{cases}\int_{x}^{t} w_{n}\left(\tau_{1}\right) \int_{x}^{\tau_{1}} w_{n-1}\left(\tau_{2}\right) \ldots \int_{x}^{\tau_{n-2}} w_{2}\left(\tau_{n-1}\right) d \tau_{n-1} \ldots d \tau_{1} & \text { for } x \leqslant t \leqslant b, \\
0 & \text { for } t<x .\end{cases}
\end{aligned}
$$

Hence $M_{i, n} \in S_{\Delta}^{n}[a, b]$.

Let $M_{j, n-1}$ be the basic spline w.r.t. the operator $\tilde{L}_{n-1}=D_{0} D_{n} \ldots D_{2}$ (all points of multiplicity $n+1$ are exchanged for points of multiplicity $n$ ). Put

$$
\tilde{M}_{j, n-1}(x)=M_{j, n-1}(x) / \int_{i_{j}}^{t_{j+n}} w_{1}(\tau) M_{j, n-1}(\tau) d \tau \text {. }
$$


Definition 5. The $j$ th normalized $L_{n} B$-spline w.r.t. the partition $\Delta$ is defined by

(6) $\quad N_{j, n}(x)$

$$
= \begin{cases}\chi_{t_{j}}(x)-\int_{a}^{x} w_{1}(\tau) \tilde{M}_{j+1, n-1}(\tau) d \tau & \text { for } t_{j}=\ldots=t_{j+n}<t_{j+n+1}, \\ \int_{a}^{x} w_{1}(\tau)\left[\tilde{M}_{j, n-1}(\tau)-\tilde{M}_{j+1, n-1}(\tau)\right] d \tau & \text { for } t_{j}<t_{j+n} \text { and } t_{j+1}<t_{j+n+1}, \\ \int_{a}^{x} w_{1}(\tau) \tilde{M}_{j, n-1}(\tau) d \tau-\chi_{i_{j+n}}(x) & \text { for } t_{j}<t_{j+1}=\ldots=t_{j+n+1},\end{cases}
$$

where $\chi_{t}(x)=1$ for $x>t$ and 0 for $x \leqslant t$, and for $j=-n$ we put $N_{-n, n}(a)$ $=1$.

Again by (5) we obtain $N_{j, n} \in S_{\Delta}^{n}[a, b]$.

Basic splines w.r.t. the Tchebyshev systems were defined by Karlin in [4] and in the special case of trigonometric splines by Schoenberg in [9]. Normalized basic splines were defined in another way by Marsden in [6]. With the help of Definition 5 we shall obtain a few further properties of basic splines analogous to polynomial splines. [1], [2])

2. Properties of basic splines. For polynomial $B$-splines we have (see

$$
\left[t_{0}, \ldots, t_{n+1} ; f\right]=\frac{1}{(n+1) !} \int_{t_{0}}^{t_{n+1}} f^{(n+1)}(t) \hat{M}_{0, n}(t) d t
$$

where $\hat{M}_{0, n}$ is the $B$-spline w.r.t. the system $\left\{t^{i}\right\}_{0}^{n}$. Since $\int_{t_{0}}^{t_{n+1}} \hat{M}_{0, n}(t) d t=1$ (see [1], [2]), we have

$$
\lim _{t_{n+1} \rightarrow t_{0}}\left[t_{0}, \ldots, t_{n+1} ; f\right]=\frac{1}{(n+1) !} f^{(n+1)}\left(t_{0}\right) .
$$

By (3) and (4) we obtain

$$
\int_{I_{0}}^{t_{n+1}} M_{0, n}(t) d t=1
$$

As for polynomial splines, we apply the generalized Taylor formula for Tchebyshev systems (see [1], [2], [5]) and obtain

$$
\left[t_{0}, \ldots, t_{n+1} ; f\right]_{*}=\int_{t_{0}}^{t_{n+1}} L_{n}^{*} f(t) M_{0, n}(t) d t .
$$


Hence

$$
\lim _{t_{n+1} \rightarrow t_{0}}\left[t_{0}, \ldots, t_{n+1} ; f\right]_{*}=L_{n}^{*} f\left(t_{0}\right)
$$

Applying (7) and the properties of determinants, we obtain

TheOrem 1. Let $\Delta^{\prime}=\left\{a \leqslant t_{-n}^{\prime}<t_{-n+1}^{\prime}<\ldots<t_{N+n}^{\prime} \leqslant b\right\}$ be a partition of the interval $[a, b]$ with distinct points and let $f \in C^{n+1}[a, b]$. Then there exists

$$
\lim _{\substack{t_{i}^{\prime} \rightarrow t_{i} \\ i=0, \ldots, n+1}}\left[t_{0}^{\prime}, \ldots, t_{n+1}^{\prime}: f\right]_{*}=\left[t_{0}, \ldots, t_{n+1}: f\right]_{*} .
$$

Hence it follows that it suffices to prove the properties of splines for partitions with distinct points.

Further we need the following

LEMmA 2. Every spline $\varphi \in S_{\Delta_{j}}^{n}[a, b]$ satisfying the conditions

$$
D^{i} \varphi\left(t_{k}\right)=0, \quad i=0, \ldots, n-1, k=j, j+n+1,
$$

can be represented in the form

$$
\varphi(x)=\alpha M_{j, n}(x),
$$

where $\alpha$ is a constant depending only on the function $\varphi$ and $\Delta_{j}=\left\{t_{j}<\ldots\right.$ $\left.<t_{j+n+1}\right\}$.

Proof. The partition $\Delta_{j}$ has only distinct points. Since $w_{j}>0$, the function $\varphi$ satisfies the following conditions:

$$
L_{i} \varphi\left(t_{k}\right)=0, \quad k=j, j+n+1, i=-1,0, \ldots, n-2,
$$

where $L_{0} \varphi=D_{0} \varphi$ and $L_{-1} \varphi=\varphi$. Put -

$$
\begin{aligned}
& \tilde{\varphi}_{n-i}(t, x) \\
& = \begin{cases}\int_{i}^{x} w_{n}\left(\tau_{1}\right) \int_{\tau_{1}}^{x} w_{n-1}\left(\tau_{2}\right) \ldots \int_{\tau_{n-i-1}}^{x} w_{i+1}\left(\tau_{n-i}\right) d \tau_{n-i} \ldots d \tau_{1} & \text { for } t \leqslant x, \\
0 & \text { for } x<t,\end{cases}
\end{aligned}
$$

$i=-1,0, \ldots, n-2$.

We can write the function $\varphi$ in the following form (see [5]):

$$
\varphi(x)=\sum_{l=j}^{j+n} b_{l} \tilde{\varphi}_{n}\left(t_{l}, x\right)
$$

Therefore

$$
L_{i} \varphi\left(t_{i+n+1}\right)=\sum_{i=j}^{j+n} b_{l} \tilde{\varphi}_{n-i-1}\left(t_{l}, t_{j+n+1}\right), \quad i=-1,0, \ldots, n-2 .
$$

This system has the matrix $A_{n}=\left[\tilde{\varphi}_{i}\left(t_{l}, t_{j+n+1}\right), i=n, \ldots, 1, l=j, \ldots, j+n\right]$. 
Let $B_{n}$ be the matrix obtained from $A_{n}$ by concelling the last column of $\underset{t_{j+1}}{A_{n}}$. We shall prove that $\operatorname{det} B_{n}>0$. For $n=1, B_{1}=\tilde{\varphi}_{1}\left(t_{j}, t_{j+1}\right)$ $=\int_{t_{j}} w_{1}(t) d t>0$, because $w_{1}>0$. Assume that $\operatorname{det} B_{n-1}>0$ for every system of functions $\left\{\tilde{w}_{i}\right\}_{0}^{n-1}, \tilde{w}_{i}>0$. Subtracting the $i$ th column from its predecessor and factoring out the integrals from the function $w_{n}$, we obtain $B_{n}=\int_{t_{j}}^{t_{j+1}} w_{n}\left(x_{1}\right) \ldots \int_{t_{j+n-2}}^{t_{j+n-1}} w_{n}\left(x_{n-1}\right) \int_{t_{j+n-1}}^{t_{j+n+1}} w_{n}\left(x_{n}\right) \tilde{B}_{n-1}\left(x_{1}, \ldots, x_{n}\right) d x_{1} \ldots d x_{n}$, where $\tilde{B}_{n-1}\left(x_{1}, \ldots, x_{n}\right)=\operatorname{det}\left[\tilde{\varphi}_{n-i}\left(x_{1}, t_{j+n+1}\right), i=1, \ldots, n, l=1, \ldots, n\right]$.

Repeating this reasoning for the determinant $\tilde{B}_{n-1}$ and expanding the determinant w.r.t. the last row, we obtain

$$
\begin{aligned}
\operatorname{det} B_{n}= & \int_{i_{j}}^{t_{j+1}} w_{n}\left(x_{1}\right) \ldots \int_{t_{j+n-2}}^{i_{j+n-1}} w_{n}\left(x_{n-1}\right) \int_{t_{i+n-1}}^{t_{j+n+1}} w_{n}\left(x_{n}\right) \int_{x_{1}}^{x_{2}} w_{n-1}\left(y_{1}\right) \ldots \\
& \ldots \int_{x_{n-1}}^{x_{n-1}} w_{n-1}\left(y_{n-1}\right) B_{n-1}\left(y_{1}, \ldots, y_{n-1}\right) d x_{1} \ldots d x_{n} d y_{1} \ldots d y_{n-1},
\end{aligned}
$$

where $B_{n-1}\left(y_{1}, \ldots, y_{n-1}\right)=\operatorname{det}\left[\tilde{\varphi}_{n-i}\left(y_{l}, t_{j+n+1}\right), i=2, \ldots, n, l=1, \ldots, n-1\right]$. Since $x_{i}<x_{1+1}, i=1, \ldots, n$, we have $B_{n-1}>0$ by the inductive hypothesis, whence det $B_{n}>0$ and the theorem is proved.

Corollary. There exists a constant $r_{j}$ such that

$$
N_{j, n}(x)=r_{j} M_{j, n}(x) .
$$

Remark. For the system $\left\{x^{i}\right\}_{0}^{n}, r_{j}=\left(t_{j+n+1}-t_{j}\right) /(n+1)$.

THEOREM 2. There exists a constant $C$ depending only on the system $\left\{u_{i}\right\}$ such that

$$
C^{-1} \hat{M}_{j, n}(x) \leqslant M_{j, n}(x) \leqslant C \hat{M}_{j, n}(x),
$$

where $\hat{M}_{j, n}$ is the $j$-th $B$-spline for the system $\left\{x^{i}\right\}_{0}^{n}$.

Proof. Assume that $\Delta$ has only distinct points.

$$
M_{j, n}(x)=D\left(\begin{array}{l}
t_{0}, \ldots, t_{n}, t_{n+1} \\
v_{0}, \ldots, v_{n}, \varphi_{n}(t, x)
\end{array}\right) / D\left(\begin{array}{l}
t_{0}, \ldots, t_{n}, t_{n+1} \\
v_{0}, \ldots, v_{n}, w
\end{array}\right)=L_{n}(x) / M_{n}(x)
$$

and analogously $\hat{M}_{j, n}(x)=\hat{L}_{n}(x) / \hat{M}_{n}(x)$.

$$
\begin{aligned}
& L_{n}(x)=\operatorname{det}\left[a_{i j}\right]_{i, j=0}^{n+1}, \text { where } a_{0 j}=1, j=0, \ldots, n+1, \\
& a_{i j}=\int_{a}^{t_{j}} w_{n}\left(\tau_{1}\right) \int_{a}^{\tau_{1}} w_{n-1}\left(\tau_{2}\right) \ldots \int_{a}^{\tau_{n-i}} w_{n-1+1}\left(\tau_{i}\right) d \tau_{i} \ldots d \tau_{1}, \\
& i=1, \ldots, n, j=0, \ldots, n+1, \\
& a_{n+1, j}=\varphi_{n}\left(t_{j}, x\right)=\int_{a}^{j} w_{n}\left(\tau_{1}\right) \int_{a}^{\tau_{1}} w_{n-1}\left(\tau_{2}\right) \ldots \int_{a}^{\tau_{n-1}} w_{1}\left(\tau_{n}\right) \varrho_{x}\left(\tau_{n}\right) d \tau_{n} \ldots d \tau_{1},
\end{aligned}
$$

$j=0, \ldots, n+1$, where $\varrho_{x}(t)=1$ for $t \geqslant x$ and 0 for $t<x$. 
Subtracting the $j$ th column from its successor, then expanding the determinant w.r.t. the first row and applying the properties of determinants, we obtain

$$
\begin{array}{r}
L_{n}(x)=\int_{i_{0}}^{t_{1}} w_{n}\left(x_{1}\right) \ldots \int_{i_{n}}^{i_{n+1}} w_{n}\left(x_{n+1}\right) \operatorname{det}\left[b_{i j}\right] d x_{1} \ldots d x_{n+1}, \\
\text { where } b_{0 j}=1, j=1, \ldots, n+1, \\
b_{i j}=\int_{a}^{x_{j}} w_{n-1}\left(\tau_{1}\right) \int_{a}^{\tau_{1}} w_{n-2}\left(\tau_{2}\right) \ldots \int_{a}^{\tau_{i-1}} w_{n-i}\left(\tau_{i}\right) d \tau_{i} \ldots d \tau_{1}, \\
\quad \text { for } b_{n+1, j}=\int_{a}^{x_{j}} w_{n-1}\left(\tau_{1}\right) \int_{a}^{\tau_{1}} w_{n-2}\left(\tau_{2}\right) \ldots \int_{a}^{\tau_{n-2}} w_{1}\left(\tau_{n-1}\right) \varrho_{x}\left(\tau_{n-1}\right) d \tau_{n-1} \ldots d \tau_{1}, \\
j=1, \ldots, n+1 .
\end{array}
$$

Repeating this reasoning, we obtain

$$
\begin{aligned}
L_{n}(x)= & \int_{t_{0}}^{t_{1}} w_{n}\left(x_{1,1}\right) \ldots \int_{t_{n}}^{t_{n+1}} w_{n}\left(x_{1, n+1}\right) \int_{x_{1,1}}^{x_{1,2}} w_{n-1}\left(x_{2,1}\right) \ldots \\
& \ldots \int_{x_{1, n}}^{x_{1, n+1}} w_{n-1}\left(x_{2, n}\right) \int_{x_{2,1}}^{x_{2,2}} w_{n-2}\left(x_{3,1}\right) \ldots \\
& \ldots \int_{x_{n-1,1}}^{x_{n-1,2}} w_{1}\left(x_{n, 1}\right) \int_{x_{n-1,2}}^{x_{n-1,3}} w_{1}\left(x_{n, 2}\right)\left[\varrho_{x}\left(x_{n, 2}\right)-\right. \\
& \left.-\varrho_{x}\left(x_{n, 1}\right)\right] d x_{1,1} \ldots d x_{1, n+1} d x_{2,1} \ldots d x_{n, 1} d x_{n, 2} .
\end{aligned}
$$

Since $x_{j, k} \leqslant x_{j, k+1}$, the difference $\varrho_{x}\left(x_{n, 2}\right)-\varrho_{x}\left(x_{n, 1}\right)$ admits only two values, 0 and 1. Remember that, for the system $\left\{x^{i}\right\}_{0}^{n}, w_{0}=1, w_{i}=i, i=1, \ldots, n$. Since the functions $w_{i}$ are continuous and positive in the interval $[a, b]$, there exist positive constants $c_{i}$ and $d_{i}$ such that

$$
0<i c_{i} \leqslant w_{i} \leqslant i d_{i} \quad \text { for } i=1, \ldots, n \text {. }
$$

Writing $\hat{L}_{n}(x)$ in the form (9), we obtain

(11) $\hat{L}_{n}(x)$

$$
=2^{3} 3^{4} \ldots n^{n+1} \int_{t_{0}}^{t_{1}} \ldots \int_{t_{n}}^{t_{n+1}} \int_{x_{1,1}}^{x_{1,2}} \ldots \int_{x_{n-1,2}}^{x_{n-1,3}}\left[\varrho_{x}\left(x_{n, 2}\right)-\varrho_{x}\left(x_{n, 1}\right)\right] d x_{1,1} \ldots d x_{n, 2} .
$$

Since $M_{n}(x)=\int_{t_{0}}^{t_{n+1}} L_{n}(x)$ and $\hat{M}_{n}(x)=\int_{t_{0}}^{t_{n+1}} \hat{L}_{n}(x) d x$, we have by (9)-(11)

$$
\begin{aligned}
\left(\frac{c_{1}}{d_{1}}\right)^{2}\left(\frac{c_{2}}{d_{2}}\right)^{3} \cdot \ldots \cdot\left(\frac{c_{n}}{d_{n}}\right)^{n+1} \hat{M}_{j, n}(x) & \leqslant M_{j, n}(x) \\
& \leqslant\left(\frac{d_{1}}{c_{1}}\right)^{2}\left(\frac{d_{2}}{c_{2}}\right)^{3} \cdot \ldots \cdot\left(\frac{d_{n}}{c_{n}}\right)^{n+1} \hat{M}_{j, n}(x) .
\end{aligned}
$$

25 - Annales Polonici Mathematici XLVI 
Since the above constants depend only on the functions $w_{j}$, by Theorem 1 we obtain the theorem under consideration.

THEOREM 3. Basic splines have the following properties:

(M.1) $\operatorname{supp} M_{j, n}=\left[t_{j}, t_{j+n+1}\right], \quad M_{j, n}(x)>0 \quad$ for $t_{j}<x<t_{j+n+1}$,

(N.1) $\operatorname{supp} N_{j, n}=\left[t_{j}, t_{j+n+1}\right], \quad N_{j, n}(x)>0$ for $t_{j}<x<t_{j+n+1}$,

$$
\begin{gathered}
\int_{i_{j}}^{j^{j+n+1}} M_{j, n}(x) d x=1, \\
\sum_{j=-n}^{N-1} N_{j, n}(x)=1 .
\end{gathered}
$$

Proof. The first three properties follow from the definition of basic splines, Lemma $1,(8)$ and the properties of polynomial splines. Let $x \in\left[t_{k}, t_{k+1}\right]$. Then

$$
\begin{aligned}
\sum_{j=-n}^{N-1} N_{j, n}(x) & =\sum_{j=k-n}^{k} N_{j, n}(x) \\
& =\int_{a}^{x} w_{1}(\tau) \sum_{j=k-n}^{k}\left[\hat{M}_{j, n-1}(\tau)-\tilde{M}_{j+1, n-1}(\tau)\right] d \tau \\
& =\int_{a}^{x} w_{1}(\tau)\left[\tilde{M}_{k-n, n-1}(\tau)-\tilde{M}_{k+1, n-1}(\tau)\right] d \tau \\
& =\int_{a}^{x} w_{1}(\tau) \tilde{M}_{k-n, n-1}(\tau) d \tau=1
\end{aligned}
$$

for $t_{j}<t_{j+n}$ and $t_{j+1}<t_{j+n+1}$. To the remaining cases of (N.2) we apply Theorem 1.

Now, we estimate the constants $r_{j}, r_{j}=N_{j, n}(x) / M_{j, n}(x), x \in\left(t_{j}, t_{j+n+1}\right)$. Hence for $x \in\left(t_{j}, t_{j+1}\right)$ by (12) we obtain

$$
r_{j}=\frac{\int_{i_{j}}^{x} w_{1}(\tau) M_{j, n-1}(\tau) d \tau}{M_{j, n}(x) \int_{t_{j}}^{t_{j+n+1}} w_{1}(\tau) M_{j, n-1}(\tau) d \tau} \leqslant(n+1) \beta_{n} \frac{\hat{N}_{j, n}(x)}{\hat{M}_{j, n}(x)}=\left(t_{j+n+1}-t_{j}\right) \beta_{n}
$$

and analogously $r_{j} \geqslant \alpha_{n}\left(t_{j+n+1}-t_{j}\right)$, where $\alpha_{n}$ and $\beta_{n}$ depend only on the system $\left\{u_{i}\right\}$ and $\hat{N}_{j, n}$ is the $j$ th normalized $B$-spline for the system $\left\{x^{i}\right\}_{0}^{n}$. Thus we have proved

THEOREM 4. There exist constants $\alpha_{n}$ and $\beta_{n}$ depending only on the system $\left\{u_{i}\right\}_{0}^{n}$ such that

$$
\alpha_{n}\left(t_{j+n+1}-t_{j}\right) \leqslant r_{j} \leqslant \beta_{n}\left(t_{j+n+1}-t_{j}\right)
$$


From (6) we obtain

$$
\begin{aligned}
& \text { Lemma } 3 \text {. Put } \beta_{j}=\left(\int_{t_{j}}^{t_{j+n+1}} w_{1}(\tau) M_{j, n-1}(\tau) d \tau\right)^{-1} \text {. Then } \\
& D_{1} N_{j, n}= \begin{cases}\beta_{j} M_{j, n-1} & \text { for } t_{j+1}=t_{j+n+1}, \\
\beta_{j} M_{j, n-1}-\beta_{j+1} M_{j+1, n-1} & \text { for } t_{j}<t_{j+n} \text { and } t_{j+1}<t_{j+n+1}, \\
-\beta_{j+1} M_{j+1, n-1} & \text { for } t_{j}=t_{j+n} .\end{cases}
\end{aligned}
$$

Hence follows

Theorem 5. Let $f \in S_{\Delta}^{n}[a, b]$ and $f=\sum_{j=-n}^{N-1} a_{j} N_{j, n}$. Then

$$
\begin{aligned}
D_{1} f=-a_{-n \beta-n+1} & M_{-n+1, n-1}+ \\
& +\sum_{\substack{j=-n+2 \\
t_{j}<t_{j+n}}}^{N-2}\left(a_{j}-a_{j-1}\right) \beta_{j} M_{j, n-1}+a_{N-1} \beta_{N-1} M_{N-1, n-1} .
\end{aligned}
$$

Hence, as for polynomial splines, we obtain

Corollary. The system $\left\{N_{j, n}\right\}_{-n}^{N-1}$ is a basis in the space $S_{\Delta}^{n}[a, b]$.

Put $N_{j, n, p}=r_{j}^{-1 / p} N_{j, n}, 1 \leqslant p \leqslant \infty$. As for polynomial splines, we can prove (see [2], [8], [10])

THEOREM 6. There exists a constant $D_{n}>0$ depending only on the system $\left\{u_{i}\right\}$ such that

$$
D_{n}\|a\|_{p} \leqslant\left\|\sum_{j=-n}^{N-1} a_{j} N_{j, n, p}\right\|_{p} \leqslant\|a\|_{p}
$$

where $a=\left(a_{-n}, \ldots, a_{N-1}\right) \in l_{p}, 1 \leqslant p \leqslant \infty$.

From Theorems 5 and 6 we obtain

Corollary. There exist constants $C_{1}$ and $C_{2}$ depending only on $p$ and $q$ $(1 \leqslant p, q \leqslant \infty)$ and the system $\left\{u_{i}\right\}$ such that for any $s \in S_{\Delta}^{n}[a, b]$

$$
\|D s\|_{p} \leqslant C_{1}\|s\|_{p}\left[\min \left(t_{j+n}-t_{j}\right)\right]^{-1}
$$

and

$$
\|s\|_{p} \leqslant C_{2}\|s\|_{q} \max _{j}\left[\left(t_{j+n+1}-t_{j}\right)^{1 / q-1 / p}\right] .
$$

3. L-splines. Let

$$
L=D^{n+1}+\sum_{i=0}^{n} a_{i}(x) D^{i}
$$

be a linear differential operator defined on the interval $[a, b]$ with the null space $N_{L}$. 
Definition 6. A function $s$ is called an $L$-spline w.r.t. the partition $\Delta$ if

$$
\begin{aligned}
& \text { (a) } L s=0 \text { on the intervals } \quad\left(s_{j-1}, s_{j}\right), j=1, \ldots, M \text {, } \\
& \text { (b) } \exists \varepsilon_{j}>0: s \in C^{n-\alpha_{j}}\left(s_{j}-\varepsilon_{j}, s_{j}+\varepsilon_{j}\right), \quad j=1, \ldots, M-1 \text {, }
\end{aligned}
$$

where $\alpha_{j}$ is the multiplicity of the point $s_{j}$.

We can reduce the investigation of $L$-splines to the investigation of Tchebyshev splines by means of the following theorem:

TheOREM 7 (see [5]). For every operator $L$ of the form (13) there exists $\delta>0$ such that, for every subinterval $I \subset[a, b]$ with the length $|I| \leqslant \delta$, the space $N_{L}$ has a basis $\left\{u_{i}^{I}\right\}_{0}^{n}$ which is an ECT-system in the subinterval $I$.

Assume that $\max \left(t_{i+n+1}-t_{i}\right)<\delta / 4$ and $a_{0}(x)=0$. Then the definition and properties of basic splines reduce to those of Tchebyshev splines. If there exists a function $u(x)>0$ for $x \in[a, b]$ in the space $N_{L}$, then we can change the basis $\left\{u_{i}\right\}_{0}^{n}$ of the space $N_{L}$ for the system which includes 1 . In the general case we divide the interval $[a, b]$ into a finite number of subintervals $I_{j}$ with the length $\delta / 4<\left|I_{j}\right|<\delta / 2$ and we repeat the above construction of $L_{n} B$-splines, in each of the subintervals $J_{i}=I_{i} \cup I_{i+1}$.

\section{References}

[1] C. De Boor, Splines as linear combination of B-splines, 1-47, Approximation Theory II, Ed. by G. G. Lorentz, C. K. Chui, L. L. Schumaker, Acad. Press, New York 1976.

[2] Z. Ciesielski, Teoria funkcji giętych (wyklad monograficzny), Uniwersytet Gdański 1976/1977.

[3] A. O. Gelfond, Iscislenie koneznyh raznostei, Fizmatgiz, Moskva 1959.

[4] S. Karlin Total positivity, Stanford University Press, Stanford, California, 1968.

[5] -, W. J. Studden, Tchebycheff systems: with applications in analysis and statistics, Interscience Publishers, New York 1966.

[6] M. Marsden, An identity for spline functions with applications to variation-diminishing spline approximation, J. Approx. Th. 3 (1970), 7. 49.

[7] G. Muhlbach, A recurrence formula for generalized divided differences and some applications, ibidem 9 (1973), 165-172.

[8] K. Scherer, L. L. Schumaker, A dual basis for L-splines and applications, ibidem 29 (1980), 151-169.

[9] I. J. Schoenberg, On trigonometric spline interpolation, J. Math. Mech. 13 (1964), 795825.

[10] L. L. Schumaker, Spline functions: basic theory, Wiley and Sons, New York 1981. 